\title{
Index rerum ad Vol. 20
}

\section{Confecit Wilhelm}

Acido nalidixico, 12 Adenomatoid tumors, 91 Angio-renographic studies in rats, 246 Aspiratio suprapubica, 1 Aspirations-biopsy of testis, 176 Bladder, electrical stimulation, 1 -, reticulum cell sarcoma, 29 -, retroperotoneal fibrosis, 29 -, Thio-Tepa in Papillomatosis, 23 -, traumatic cord, 1 Bladder tumeurs, histochemical study,42 - -, urinary Lactate-dehydrogenase, 359 Blase, retroperitoneale Fibrose, 29 -, Retothelsarkom, 29 -, traumatische Rückenmarksblase, 1 Blasentumoren, Behandlung mit Thio-Tepa, 23 -, Beta-Glucuronidasebestimmungen, 206 -, histochemische Untersuchungen, 42 -, Urin-Lactatdehydrogenase, 359 Bricker-Operation, 1 Calicolitotomia, 255 Càlculos de la prostata, prostatectomia transuretral, 319 Carcinome de la prostate, implants d'hormones, 289 Cystinuria, 154 Cystostomy, catheterless, 1 Dehydrogenase systems in bladder tumeurs, 42 Deisting, dilatatio prostatae, 17 Dilatation et prostatectomie, 17 Double-rein chez Гenfant, 100 Electrical stimulation of the bladder, 1 Epididyme, tumeurs adénomateuses, 91 Estrogen therapy in prostatic carcinoma,

289 Fibrose retroperitoneale de la vessie, 29 Fosfatasa en los tumores de vejiga, 42 Glucuronidase y tumores vesicales, 206 Harnwegsinfektionen, Behandlung der therapieresistenten, 143

i ad Vol. 20

Baumann, Zurich

Hipertrofia prostàtica, dilatación, 17

-, la función renal, 191

-, resección transuretral, 17 Hoden, Aspirationsbiopsie, 176 Hoden, hormonale Untersuchungen nach Orchidektomie, 364

Hodentumoren, Behandlung, 129

Honvan,289

Hyperparathyreoidism, electron microscopic study on the kidney, 319

Immunoélectrophorèse avant et après prostatectomie, 65

Induratio penis plastica, therapia, 236

Infections of the urinary passages, treatment, 143

Infections urinaires résistantes, traite-ment, 143

Kidney, double-kidney in the infant, 100

Lactate-dehydrogenase in bladder tumors, 359

Lithiase rénale, conceptions chirurgi-cales, 255

-, resection partielle du rein, 171

-, tuberculose, 77

Lymphatic circulation of the kidney, 305

Nalidixic acid, 12

Nebenhoden, adenomatoîd-Tumoren,91

Nephrolithiasis, neue chirurgische Behandlung, 255 
-, Nierentuberkulose, 77

Niere, angio-renographische Untersuchungen, 246

-, Lymphwege, 305

-, Struktur des Beckenkelchsysterns, 222

-, Studien bei Hyperparathyreoidismus, 319

Nierenlymphe, 305

Nierenpolresektion, 171

Orchidektomie, hormonale Untersuchungen, 364

Orina residual, 100

Papillomatosis, instillations of Thio-Tepa, 23

Penis, induratio plastica, 236

378

Index rerum

Phosphatase et tumeurs de la vessie, 42 Plastic induration of the penis, 236 Prostatacarcinom, implantierte Hor-

monpreßlinge, 289 Prostatahypertrophie, Dilatation, 17 -, Nierenfunktion, 191 -, transurethrale

Resektion, 17 Prostata-Steine, transurethrale Prostat-

ektomie, 319 Prostatectomie radicale transpubienne,

347 -, transurethrale pour calculs, 319 Prostatectomy, immunoelectrophoretic

studies, 65-, treatment with Trasylol, 65Prostatic hyperplasia, dilatation, 17 , renal

functions, 191

- $\quad$-, transurethral resection, 17Rein, examen angiographique, 246-, hyperparathyréoidisme, 319

-, la circulation lymphatique, 305 Renal calculosis, resection of the renal pole, 171

calyceo-pelvic system, 222

lithiasis, new surgical concepts, 255

-, tuberculosis, 77

lymphatics, 305 Renographische Untersuchungen bei

Ratten, 246

Resection transurethrale et la prostatectomie, 17

Residual urine, 117

Reticulum cell sarcoma of the bladder, 29

Rétothelsarcome de la vessie, 29

Retroperitoneal fibrosis of the bladder, 29

Riñon, duplicidad en el niño, 100

-, examen angiográfico, 246

-, hiperparatiroidismo experimental,319

-, los linfaticos, 305

-, resección parcial, 171

-, sistema pielocalicial, 222

-, tuberculosis-litiasis, 77

Rückenmarksblase, traumatische, 1

Seminoma, 129

Suprapubic aspiration, 1

Suprapubic cystostomy, 1 
Testicular carcinoma, 129

Testicule, l'orchidectomie et l'élimina-

tion hormonale, 364 Testiculo, biopsia con aguja aspiradora,

176 -, tratamiento de los tumores, 129 Thio-Tepa in papillomatosis of the

bladder, 23 Transpubic radical prostatectomy, 347 Transurethral prostatectomy for lithiasis, 336

-, resection, 17

Traumatic cord bladder, management, 1 Trasylol après prostatectomie, 65 Tuberculose, lithiase rénale, 77 Tumeurs du testicule, traitement, 129 Urine résiduelle, 117 Urologia neurologica, 1 Vasectomia prophylactica, 163 Vejiga, estudio histoquimico de los

tumores, 42 - neurógena traumatica, 1 Vesical Neoplasia et Beta-Glucuronid-

ase, 206 Vesicostomia, paralysia traumatica, 1 Vessie du traumatisé médullaire, 1 -, Lactadehydrogenase lors de tumeur,

3. 359 -, la /î-gluconidase et les tumeurs

$206 \sim$

Гétude histochimique des tumeurs

$42 \sim$

structure du système pyélocaliciel

222 -, traitement des tumeurs par Thio-Tepa 